\title{
Unraveling the Role of Structure and Density in Determining Vertebral Bone Strength
}

\author{
J. F. Veenland, ${ }^{1,2}$ T. M. Link, ${ }^{3}$ W. Konermann, ${ }^{3}$ N. Meier, ${ }^{3}$ J. L. Grashuis, ${ }^{1,2}$ E. S. Gelsema ${ }^{1}$ \\ ${ }^{1}$ Department of Medical Informatics, Postbox 1738, Erasmus University, 3000 DR Rotterdam, The Netherlands \\ ${ }^{2}$ Department of Radiology, Erasmus University, Rotterdam, The Netherlands \\ ${ }^{3}$ Department of Clinical Radiology, Münster, Germany
}

Received: 9 July 1996 / Accepted: 24 March 1997

\begin{abstract}
The strength of bone is determined not only by bone density but also by structure. Therefore, quantification of the structure in radiographs by texture parameters may result in a better prediction of fracture risk. Since in radiographs density and structure are strongly correlated, the predictive power of texture parameters should be corrected for the influence of BMD to determine the additional information conveyed by these parameters. In this study, we evaluated the predictive power of various texture parameters based on the Grey-Level Dependence Method and the Morphological Gradient Method. This study was performed on 67 vertebrae obtained from 20 male and 12 female human cadaver thoracolumbar spines. BMD and area of the vertebral body were determined from QCT images and texture parameters were derived from direct magnification (DIMA) radiographs. The fracture force, measured under conditions simulating the in vivo situation, was corrected with the area of the vertebra to yield the fracture stress (FS). Results of the study indicate that BMD correlates significantly with FS $r=0.82(P<0.001, n=24)$ and $r=0.94(P<0.001, n$ $=43$ ) for female and male vertebrae, respectively. Correlation coefficients of the investigated texture parameters were as high as $0.80(P<0.001)$ and $0.67(P<0.001)$ for the female and male vertebrae, respectively. Multiple regression analysis showed that in female vertebrae, the addition of one texture parameter to BMD results in a better prediction of strength. The multiple correlation coefficient was $0.87(P<0.001)$ in this case. In male vertebrae, BMD was the best predictor of fracture stress. These results suggest that texture parameters, as measured in magnification radiographs, can predict bone strength. Whereas in all cases BMD is the best single predictor of bone strength, for women texture parameters contain useful additional information.
\end{abstract}

Key words: Vertebral strength - Magnification radiographs - Texture - Quantitative computed tomography.

The strength of bone is determined by bone density and structure. Whereas the density has been studied as a major determinant for years, the importance of structure has only been recognized more recently. This can be attributed to the fact that structure constitutes a feature that is, compared to density, difficult to quantify. Several sophisticated techniques have become available to estimate bone density; methods that measure structure in vivo, are being developed. Several studies [1-5] have shown that bone structure, more specifically trabecular architecture, can be quantified using texture analysis methods on radiographs.

The trabecular structure, as projected on a radiograph, has been studied using fractal dimensions $[1,2,4,5]$ and parameters derived from the Fourier Spectrum [3]. We found that several fractal dimension estimation methods are influenced by the radiographic process and therefore should only be used under certain restrictions [6]. In the present study, we have used texture features based on the Spatial Grey-Level Dependence Method (SGLDM) and on the Morphological Gradient Method. In the first method, greylevel differences over a short distance in a given direction are quantified. The texture parameters based on morphological gradients are sensitive to differences in direction, size, and shape of structures present in the image. The parameters, generated by either method, are true texture features; they are insensitive to first-order grey-level statistics such as the average optical density.

The contribution of trabecular bone to strength can be studied most directly in vertebrae, since these consist mainly of trabecular bone. In several studies, the strength of vertebrae has been related to an estimator of bone density, namely ash density [7], DPA [8, 9], DXA [10-13], and QCT $[9,11,13-19]$. Also, histologic parameters, quantifying the structure of trabecular bone, have been used in predicting the strength of vertebrae $[20,21]$. Although Caldwell et al. [22] showed that a structural parameter, derived from the Sobel edge-enhanced image, correlated significantly with compressive strength of the vertebrae, the value of this structural parameter additional to bone density in predicting strength has not yet been studied. Since structure and density are two concepts that are closely intertwined physically and therefore strongly correlated, it is important to investigate whether structural parameters add any new information, apart from the density information structural parameters inherently contain.

In this study, we first investigated the correlation between different texture parameters, determined on macroradiographs, and the strength of vertebrae. Second, the additional value of texture parameters in predicting bone strength, independent of BMD, was evaluated for the male and female vertebrae separately.

\section{Material and Methods}

Thoracolumbar spines of 32 donors, 20 males and 12 females,
Correspondence to: J. F. Veenland at the Department of Medical Informatics 
were obtained 1 or 2 days postmortem. Average age at death of the males and females was 60.2 and 56.6 years, respectively, ranging from 54 to 72 for the males and from 48 to 75 for the females. The spines were stripped of soft tissue, leaving discs, ligaments, and joint capsules intact. Donors with metastic cancer or a history of trauma, as well as all vertebrae displaying defects on gross pathologic examination, were excluded. In this way, 67 motion segments consisting of one vertebral body, the adjacent discs, and parts of the vertebrae were harvested from these spines. Of each segment, the midvertebral QCT and area was obtained, a magnification radiograph was taken, and the fracture force was determined.

\section{Quantitative Computed Tomography}

All QCT examinations were performed using a CT scanner (Tomoscan LX, Philips) in an environment simulating the spine within the human body. Midvertebral scans with a slice thickness of 10 $\mathrm{mm}$ and $120 \mathrm{kVp}$ were obtained. BMD was measured using a standardized ellipsoid region of interest (ROI). Additionally, the area of the vertebral body in the midvertebral scan was determined.

\section{Magnification Radiography}

Direct Magnification (DIMA) radiographs of all specimens were obtained using the prototype of a microfocal X-ray tube (Microfox G10, Feinfocus Medical Systems, Garbsen, Germany). The images were taken with 6-fold magnification, a focal spot size of $60 \mu \mathrm{m}$, and $50 \mathrm{kVp}$. Computed radiography was used as an imaging system employing the storage phosphor technique (FCR-7000 storage phosphor unit, Fuji, Tokyo). The matrix size of the cassette was $1760 \times 2140$ with an element size of $200 \mu \mathrm{m} \times 200 \mu \mathrm{m}$. In Figure 1 , the DIMA radiographs of a strong (A) and weak (B) vertebra are shown.

\section{Texture Parameters}

In each DIMA radiograph, a region of interest of approximately 35 $\mathrm{mm} \times 35 \mathrm{~mm}$ was selected centrally in the vertebra. This region was digitized using a CCD-camera, and the resulting pixel size was $87.5 \mu \mathrm{m} \times 87.5 \mu \mathrm{m}$. In this study, we used two texture analysis methods: the Spatial Grey-Level Dependence Method [23] and the Morphological Gradient Method that is based on mathematical morphology [24] (see Appendix). The SGLD method obtains its independence of first-order grey-level statistics by a preprocessing step, whereas the MG method is inherently independent of linear grey-scale transformations.

The reproducibility of the different texture parameters was determined on femora [25]. Only the parameters that showed a good reproducibility on femora were used in this study.

\section{Mechanical Testing}

Bone strength was determined as described by Brinckmann et al. [16]. The fracture force was obtained using an electromechanical materials testing machine in which the specimens were loaded. The specimens were mounted in a metal cup by means of a small amount of high viscosity bone cement underneath the endplate. The upper endplate was also covered with bone cement and a stainless steel plate was mounted on it. The surface of the plate was aligned parallel to the plane of the metal cup. The load was applied on the top of the mounted specimen by means of a stamp with a flat end. Compressive force on the specimens was increased gradually. Load and compressive deformation of the specimen were recorded on an xy-plotter. The curve exhibited an almost linear increase of deformation with force. Fracture occurred when the curve deviated from this linear elastic region and was accompanied by an audible cracking and extrusion of bone marrow through the ventral and dorsal venous pathways of the vertebral body of the
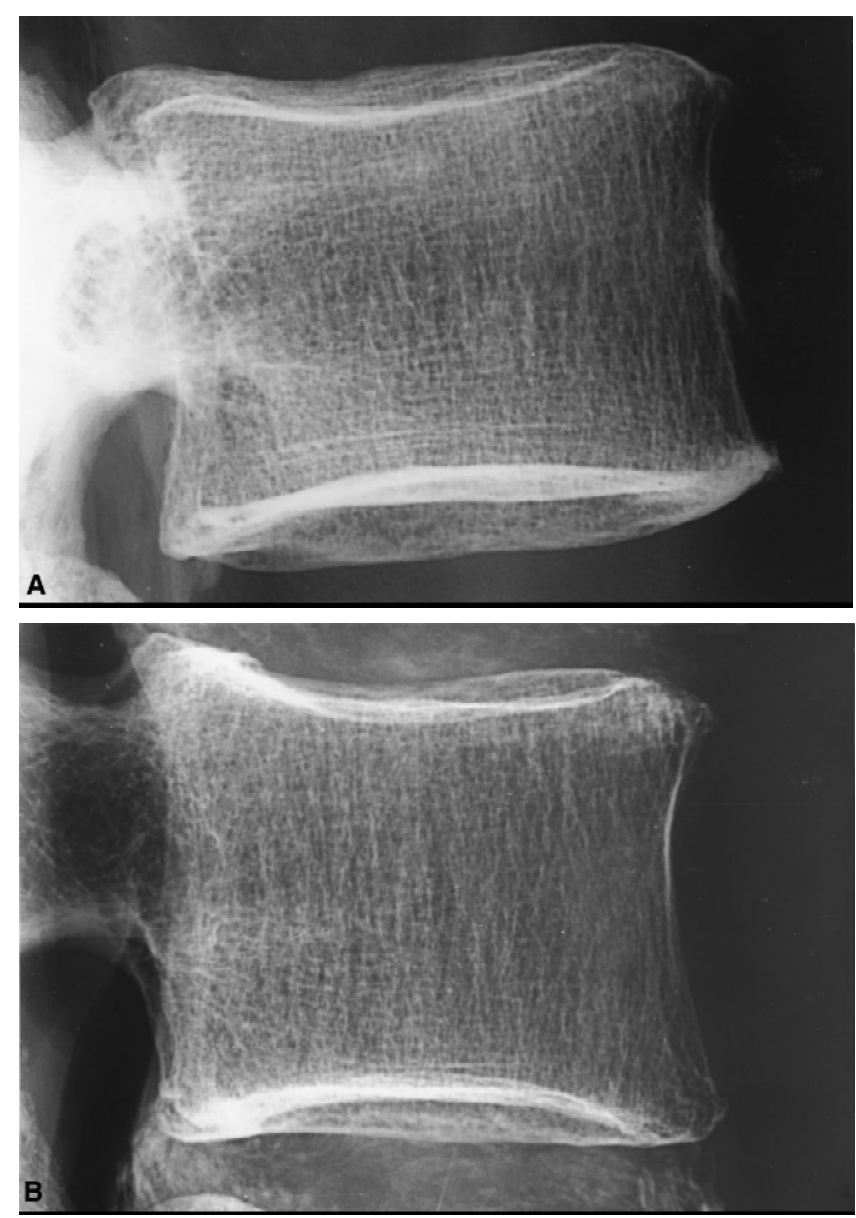

Fig. 1. Direct magnification radiographs of a strong (A) and weak (B) vertebrae.

motion segment. Fracture stress was calculated by dividing the fracture force by the midvertebral area, determined by CT. The midvertebral area was used, since the fracture load is distributed over this minimal cross-section.

\section{Statistical Analysis}

Pearson's product moment correlation coefficient was used to determine bivariate correlation coefficients. The correlation between two variables can be caused by the dependence of both variables on a third parameter. In that case, the partial correlation coefficient can be used to compute the correlation between the two variables, independent of the third parameter. When $r_{x y}, r_{x z}$, and $r_{y z}$ are the pairwise correlation coefficients between the variables $x, y$, and $z$, the partial correlation coefficient between $y$ and $z$, corrected for $x$, is given by [26]

$$
r_{y z} \cdot x=\frac{r_{y z}-r_{x y} r_{x z}}{\sqrt{\left(1-r_{x y}^{2}\right)\left(1-r_{x z}^{2}\right)}}
$$

In order to determine the correlation between a linear combination of two variables (e.g., $x$ and $y$ ) and a third parameter (e.g., $z$ ), the multiple correlation coefficient $\left(R_{z \cdot x y}\right)$ was used. The multiple correlation coefficient is defined as

$$
R_{z \cdot x y}=\sqrt{\frac{r_{x z}^{2}+r_{y z}^{2}-2 r_{x y} r_{x z} r_{y z}}{1-r_{x y}^{2}}}
$$


Table 1. BMD, area, and fracture force for the three vertebrae categories

\begin{tabular}{|c|c|c|c|c|c|c|c|c|c|c|}
\hline & \multirow[b]{2}{*}{$n$} & \multicolumn{3}{|l|}{ BMD } & \multicolumn{3}{|l|}{ Area } & \multicolumn{3}{|c|}{ Fracture Force } \\
\hline & & Mean & SD & Range & Mean & SD & Range & Mean & SD & Range \\
\hline $\mathrm{T} 11-\mathrm{T} 12$ & 26 & 135.61 & 42.48 & $62.94-215.9$ & 11.73 & 2.31 & $7.23-16.30$ & 4.94 & 1.88 & $1.50-8.35$ \\
\hline L1-L2 & 20 & 128.59 & 40.92 & $71.80-197.21$ & 12.09 & 2.22 & $7.98-16.20$ & 5.08 & 2.11 & $1.63-9.15$ \\
\hline L3-L4 & 21 & 120.78 & 37.03 & 56.97-188.09 & 12.75 & 2.23 & $8.71-15.90$ & 5.34 & 1.72 & $2.38-9.04$ \\
\hline
\end{tabular}
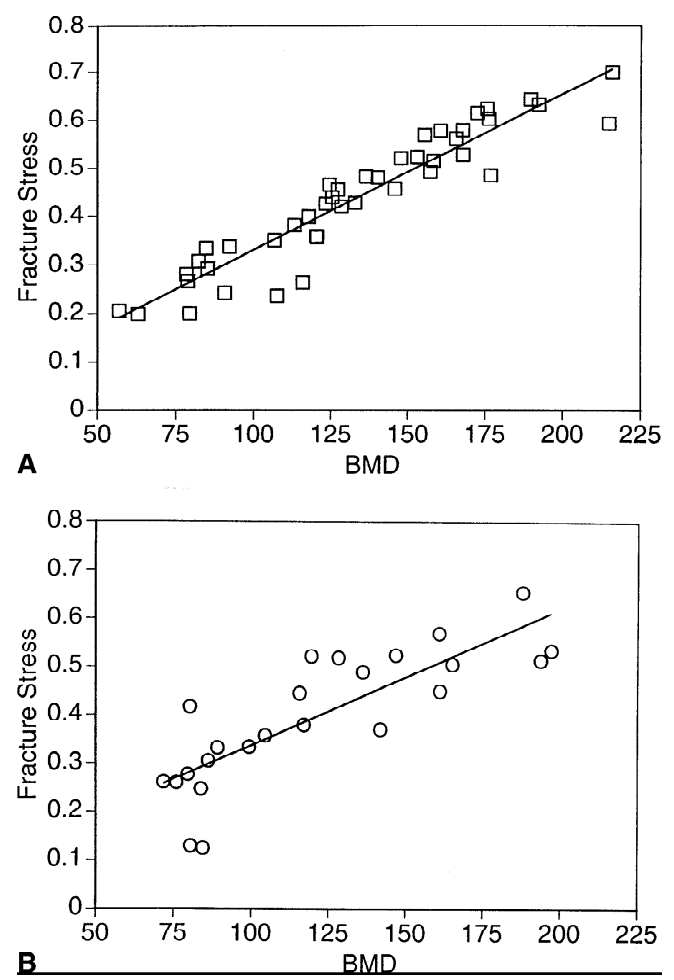

Fig. 2. Fracture stress vs. BMD for male (A) and female vertebrae (B).

The relationship between the multiple correlation coefficient $R_{z x y}$ and the partial correlation coefficient $r_{y z x}$ is described in

$$
R_{z \cdot x y}^{2}=r_{x z}^{2}+r_{y z \cdot x}^{2}\left(1-r_{x z}^{2}\right)
$$

This equation shows that the partial correlation coefficient determines whether combining the variable $y$ with $x$ will improve the prediction of parameter $z$.

In our study, we used the partial correlation coefficient for separating the contribution of BMD and structure parameters to fracture stress. Linear relations between the texture parameters, $\mathrm{BMD}$, and fracture stress were assumed. The different analyses were performed for the whole set of vertebrae, and for both sexes separately.

\section{Results}

Table 1 shows the means, standard deviations and ranges of BMD, area, and fracture force for the three vertebral categories. Since a motion segment also comprises the two adjacent vertebrae, at most one vertebra of each individual is present in each category. Whereas the BMD reaches the
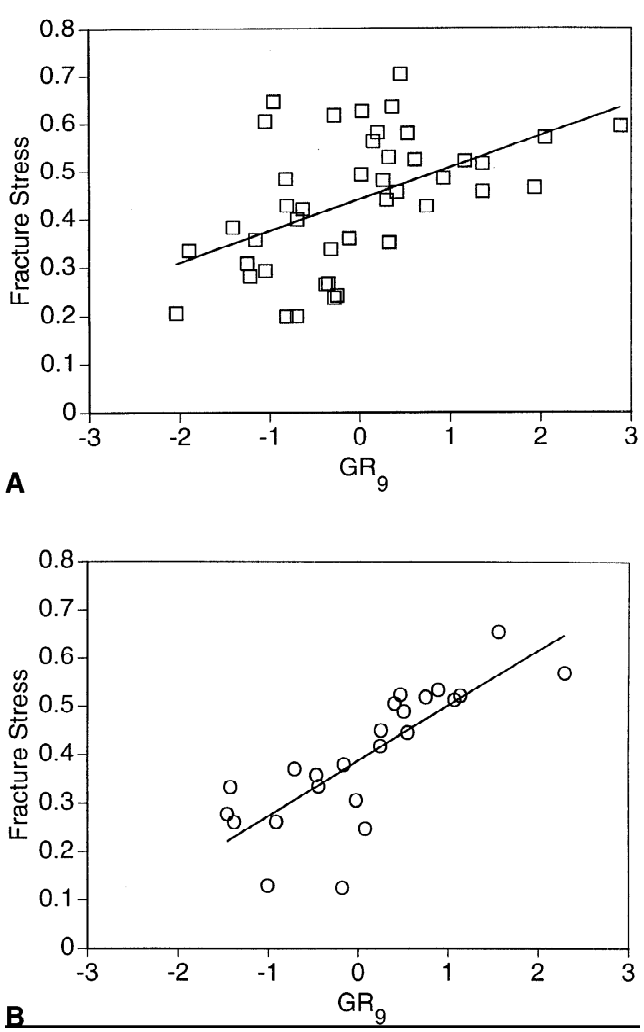

Fig. 3. Fracture stress vs. the texture parameter $\mathrm{GR}_{9}$, based on morphological gradients, for male (A) and female vertebrae (B).

highest values in category T11-T12, area and fracture force increase in the caudal direction. Fracture force is divided by area to correct for the influence of the size of the vertebra. The resulting parameter is called fracture stress.

The correlation between fracture stress and BMD for male and female vertebrae is depicted in Figures 2A and 2B, respectively. The correlation for the female vertebrae is somewhat lower than for the male vertebrae $(r=0.82$ and $r=0.94$, respectively). In Figures $3 \mathrm{~A}$ and $3 \mathrm{~B}$, the correlation between fracture stress and a texture parameter based on morphological gradients $\left(\mathrm{GR}_{9}\right)$ is illustrated. For female vertebrae, the correlation of the texture parameter with fracture stress is almost as high $(r=0.80)$ as the correlation of BMD with fracture stress, whereas in male vertebrae the correlation is lower $(r=0.50)$.

The correlation coefficients of several texture parameters with both fracture stress and BMD are listed in Tables 2, 3, and 4 for all, male, and female vertebrae, respectively. In addition, the correlation of texture parameters with fracture stress, controlling for BMD, is shown. For ease of survey, each group of texture parameters (described in the Appendix) is represented by one parameter. 
Table 2. Correlation coefficient for texture parameters with fracture stress, BMD, and fracture stress corrected for BMD for all vertebrae

\begin{tabular}{lccc}
\hline & Fracture stress & BMD & $\begin{array}{l}\text { Fracture } \\
\text { stress/BMD }\end{array}$ \\
\hline $\mathrm{COR}_{1,0^{\circ}}$ & $0.466^{\mathrm{a}}$ & $0.440^{\mathrm{a}}$ & 0.179 \\
$\mathrm{COR}_{2,0^{\circ}}$ & $0.455^{\mathrm{a}}$ & $0.419^{\mathrm{a}}$ & 0.197 \\
$\mathrm{COR}_{1,90^{\circ}}$ & $0.314^{\mathrm{b}}$ & $0.340^{\mathrm{b}}$ & 0.019 \\
$\mathrm{COR}_{2,90^{\circ}}$ & 0.141 & 0.151 & 0.012 \\
$\mathrm{GR}_{9}$ & $0.582^{\mathrm{a}}$ & $0.601^{\mathrm{a}}$ & 0.118 \\
$\mathrm{HS}_{13,9}$ & $0.697^{\mathrm{a}}$ & $0.732^{\mathrm{a}}$ & 0.128 \\
$\mathrm{HO}_{11,3}$ & $-0.437^{\mathrm{a}}$ & $-0.365^{\mathrm{b}}$ & $-0.268^{\mathrm{c}}$ \\
${ }^{\mathrm{a} P<0.001 ;{ }^{\mathrm{b}} P<0.01 ;{ }^{\mathrm{c}} P<0.05}$ & &
\end{tabular}

Table 3. Correlation coefficient for texture parameters with fracture stress, BMD, and fracture stress corrected for BMD for male vertebrae

\begin{tabular}{lccc}
\hline & Fracture stress & BMD & $\begin{array}{l}\text { Fracture } \\
\text { stress/BMD }\end{array}$ \\
\hline $\mathrm{COR}_{1,0^{\circ}}$ & $0.426^{\mathrm{b}}$ & $0.430^{\mathrm{b}}$ & 0.069 \\
$\mathrm{COR}_{2,0^{\circ}}$ & $0.420^{\mathrm{b}}$ & $0.429^{\mathrm{b}}$ & 0.051 \\
$\mathrm{COR}_{1,90^{\circ}}$ & $0.317^{\mathrm{c}}$ & $0.366^{\mathrm{c}}$ & -0.089 \\
$\mathrm{COR}_{2,90^{\circ}}$ & 0.181 & 0.241 & -0.142 \\
$\mathrm{GR}_{9}$ & $0.501^{\mathrm{a}}$ & $0.566^{\mathrm{a}}$ & -0.117 \\
$\mathrm{HS}_{13,9}$ & $0.671^{\mathrm{a}}$ & $0.727^{\mathrm{a}}$ & -0.065 \\
$\mathrm{HO}_{11,3}$ & -0.295 & -0.248 & -0.188 \\
\hline
\end{tabular}

${ }^{\mathrm{a}} P<0.001 ;{ }^{\mathrm{b}} P<0.01 ;{ }^{\mathrm{c}} P<0.05$

Table 4. Correlation coefficient for texture parameters with fracture stress, BMD, and fracture stress corrected for BMD for female vertebrae

\begin{tabular}{lccc}
\hline & Fracture stress & BMD & $\begin{array}{l}\text { Fracture } \\
\text { stress/BMD }\end{array}$ \\
\hline $\mathrm{COR}_{1,0^{\circ}}$ & $0.552^{\mathrm{b}}$ & $0.479^{\mathrm{c}}$ & 0.317 \\
$\mathrm{COR}_{2,0^{\circ}}$ & $0.540^{\mathrm{b}}$ & $0.433^{\mathrm{c}}$ & 0.358 \\
$\mathrm{COR}_{1,90^{\circ}}$ & 0.318 & 0.294 & 0.141 \\
$\mathrm{COR}_{2,90^{\circ}}$ & 0.036 & -0.073 & 0.168 \\
$\mathrm{GR}_{9}$ & $0.795^{\mathrm{a}}$ & $0.720^{\mathrm{a}}$ & $0.514^{\mathrm{c}}$ \\
$\mathrm{HS}_{13,9}$ & $0.803^{\mathrm{a}}$ & $0.781^{\mathrm{a}}$ & $0.452^{\mathrm{c}}$ \\
$\mathrm{HO}_{11,3}$ & $-0.698^{\mathrm{a}}$ & $-0.585^{\mathrm{b}}$ & $-0.470^{\mathrm{c}}$ \\
\hline
\end{tabular}

${ }^{\mathrm{a}} P<0.001 ;{ }^{\mathrm{b}} P<0.01 ;{ }^{\mathrm{c}} P<0.05$

The texture parameters based on morphological gradients, with the exception of the orientation parameter, showed a better correlation with fracture stress and BMD than the parameters based on the SGLD method. The correlation with both fracture stress and BMD was higher for the female vertebrae than for the male vertebrae. Correction for the influence of BMD, using partial regression analysis, lowered the correlation coefficients substantially. Three texture parameters, all based on morphological gradients, were still significantly correlated with fracture stress in female vertebrae, whereas in male vertebrae all correlation coefficients became insignificant. On the other hand, the correlation between BMD and fracture stress can also be corrected for the influence of texture parameters. Using $\mathrm{HS}_{13,9}$ as a texture parameter, in male vertebrae the correlation was only slightly lowered $(r=0.89, P<0.001)$, whereas in female vertebrae this correction resulted in a substantially lower partial correlation coefficient of $r=0.52(P<0.05)$.

The result of multiple regression analysis can be calculated using Eq. 3. In male vertebrae, combining BMD with texture parameters did not improve the prediction of fracture stress. In female vertebrae, however, the combination of BMD and one texture parameter resulted in higher correlations $\left(R=0.87, R=0.86, R=0.86\right.$ for $\mathrm{GR}_{9}, \mathrm{HS}_{13.9}$, and $\mathrm{HO}_{11,3}$, respectively) than for BMD alone $(r=0.82)$.

\section{Discussion}

Several studies have been performed relating density and strength of vertebrae. Due to the variety of parameters used - ash density, BMD determined by DPA, DXA, and QCT for density, ultimate force, yield strength, and absorbed energy for strength - the results of these studies are difficult to compare.

The strength of vertebrae is determined by geometry, density, and structure. The contribution of these three factors should be evaluated separately. In Table 1 it can be seen that the fracture force increases with the area of the vertebrae in the caudal direction, whereas the BMD decreases. In this study, we assumed a proportional relation between area of the vertebra and strength, so as to eliminate the influence of geometry; the fracture force is divided by the area of the vertebra, resulting in the parameter fracture stress. Texture parameters are used to quantify the structure of trabecular bone, independent of first order grey-level statistics such as the average grey-level. The way that the texture parameters are related exactly to the underlying three-dimensional structure of the trabecular bone is still unclear. We choose to evaluate the vertebrae of men and women separately, since the female vertebrae are reported to contain relatively more trabecular bone than male vertebrae [27].

Since bone mineral density and trabecular structure are two concepts that are closely intertwined-no structure without density and no density without structure-we used partial correlation techniques to investigate the influence of both factors separately. In partial correlation, the effects of other variables can be controlled by removing the linear relationship of these variables with the variables studied. In this way, in the present study, the relationship between structure and strength was studied, controlling for the effect of density. Also, the relation between density and strength was quantified independent of structure. The partial correlation coefficient will depend on the texture parameter used.

We found that the correlation between texture parameters and fracture stress was lower for male than for female vertebrae. Both in male and female vertebrae, this correlation is partly based on BMD (Tables 2, 3, and 4). After removing the influence of $\mathrm{BMD}$, only in female vertebrae the texture parameters based on morphological gradients are still significantly correlated. Therefore, it can be stated that the parameters based on the MG method, in contrast to those based on the SGLD method, are able to convey real structure information independent of BMD. Correction for the influence of the texture parameter on the correlation between BMD and fracture stress, changed the correlation coefficient for male vertebrae only slightly, whereas the correlation coefficient for female vertebrae was substantially lowered. This means that the BMD in female vertebrae is partly determined by the trabecular structure.

Multiple regression analysis showed that in male verte- 
brae BMD alone is the best predictor of fracture stress, and texture parameters do not add new information. In female vertebrae, however, a combination of one texture parameter and BMD provides a better prediction of fracture stress than BMD alone.

These observed differences between male and female vertebrae may be explained by the different ratios of trabecular and cortical bone in the vertebrae of both sexes [27]: the female vertebrae contain relatively more trabecular bone. Therefore, it can be assumed that in female vertebrae the contribution of the trabecular bone to strength is more important than in male vertebrae. Further, we conjecture that in female vertebrae the architecture of the trabecular bone is a more critical factor in determining the strength of the vertebra than in male vertebrae.

Based on these results, we can conclude that, at least in female vertebrae, trabecular structure as measured by texture parameters is an important determinant of vertebral bone strength. Moreover, we have shown that part of the structure information is already included in the BMD measurement. Therefore, structure information has additive importance to BMD in predicting strength, but because of the interrelation of structure and density, this additive value is limited.

Since the sample size in this study is fairly small, the results will have to be validated on a larger test set.

\section{Appendix}

\section{Spatial Grey-Level Dependence Method}

In 1973, Haralick [23] introduced the co-occurrence matrix and its derived parameters. This method has proven to outperform other methods in texture analysis tasks [28]. The method consists of three steps. First, a histogram equalization is performed: the number of grey-levels in the image is reduced (to $\mathrm{G}$ ) and the grey-levels are redistributed in such a way that the probability of occurrence for all grey-values is equalized. Second, a matrix $\mathbf{M}(d, \Theta)$ is composed, the elements of which are the co-occurrences of grey-levels $i$ and $j$ over a distance $d$, under an angle $\Theta$. Normalizing the matrix elements for the total number of co-occurrences yields a probability matrix $P_{i, j}$. From this matrix, as a third step a number of parameters, such as homogeneity, contrast, entropy, and correlation, can be computed.

$$
\begin{aligned}
& \operatorname{HOM}_{d, \theta}=\sum_{i, j=1}^{G, G} P_{i, j, d, \theta}^{2} \\
& \operatorname{CON}_{d, \theta}=\sum_{i, j=1}^{G, G} P_{i, j, d, \theta}(i-j)^{2} \\
& E N T_{d, \theta}=\sum_{i, j=1}^{G, G} P_{i, j, d, \theta} \log \left(P_{i, j, d, \theta}\right) \\
& \mu_{x}=R_{d, \theta}= \frac{\sum_{i, j=1}^{G, G}\left(i-\mu_{i}\right)\left(j-\mu_{j}\right) P_{i, j, d, \theta}}{G} \sigma_{x=1}^{G} \sigma_{i} \sigma_{j} \\
& \sum_{x}^{2}=\sum_{x=1}^{G}\left(x-\mu_{x}\right)^{2} \quad \text { with } \quad x=i, j
\end{aligned}
$$

We equalized the histogram to 4 grey-levels. For the distance $d$, values of 1,2 , and 4 pixels were used; for the angle $\Theta$, values of $0^{\circ}$ and $90^{\circ}$ were taken.

\section{Morphological Gradient Method}

The theory of mathematical morphology is described extensively by Serra [24]. Mathematical morphology provides techniques to enhance grey-level variations within a certain neighborhood. The size, shape, and orientation of this neighborhood is determined by the structuring element. The two basic operations - erosion and dilation-are defined using minimum and maximum filters with the neighborhood determined by the structuring element. If $f(x, y)$ is the input image, and $b(x, y)$ the structuring element, erosion $\ominus$ and dilation $\oplus$ can be defined, respectively, as

$$
\begin{gathered}
(f \ominus b)=\operatorname{MIN}\{f(s+x, t+y)-b(x, y) \mid(s+x),(t+y) \in \\
\left.D_{f} ;(x, y) \in D_{b}\right\} \\
(f \oplus b)=\operatorname{MAX}\left\{\begin{array}{c}
\text { (A.5) } \\
\left.D_{f} ;(x, y) \in D_{b}\right\}
\end{array}\right.
\end{gathered}
$$

where $D_{f}$ and $D_{b}$ are the domains of $f$ and $b$.

Several combinations of these basic operations and structuring elements are possible. For this study, we concentrated on two morphological gradients: the difference between the dilated and the eroded version of an image,

$$
g=(f \oplus b)-(f \ominus b)
$$

and the top-hat transformation,

$$
h=f-\{(f \ominus b) \oplus b)\} .
$$

By varying the shape, size, and orientation of the structuring element, different aspects of the image are enhanced. The first gradient renders the maximum variation of grey-levels within a given neighborhood, thus highlighting sharp greylevel transitions. The top-hat transformation extracts white objects from the image, the shape, size, and orientation of which are dependent on the structuring element.

We used structuring elements in the image plane. To quantify the information in the image after applying the morphological gradient, the grey-level values of the pixels in the image are summed. In order for the parameters to be insensitive for linear grey-scale changes, the results of the morphological gradients with different structuring elements are combined using ratios. In this study, the following parameters were used

$$
\begin{gathered}
G R_{i}=\frac{\sum g_{\square_{i, i}}}{\sum g_{\bigcirc_{i}}} \\
H S_{i, j}=\frac{\sum h_{\square_{i, i}}}{\sum \square_{j, j}} \\
H O_{i, j}=\frac{\sum h_{\square_{i, j}}}{\sum h_{\square_{j, i}}}
\end{gathered}
$$

where $\bigcirc_{i}$ and $\square_{i j}$ are a circular structuring element with radius $i$, and a rectangular structuring element of size $i \times j$, respectively. The first parameter, GR, is sensitive to the roundness of structures, whereas $\mathrm{HS}$ and $\mathrm{HO}$ assess size and vertical orientation of white objects present in the image. 


\section{References}

1. Lynch JA, Hawkes DJ, Buckland-Wright JC (1991) Analysis of texture in macroradiographs of osteoarthritic knees using the fractal signature. Phys Med Biol 36:709-722

2. Ruttimann ES, Webber RL, Hazelrig JB (1992) Fractal dimension from radiographs of peridental alveolar bone. Oral Surg Oral Med Oral Path 74:98-110

3. Caligiuri P, Giger ML, Favus MJ, Jia H, Doi K, Dixon LB (1993) Computerized radiographic analysis of osteoporosis: preliminary evaluation. Radiology 186:471-474

4. Benhamou CL, Lespessailles E, Jacquet G, Harba R, Jennane R, Loussot T, Tourliere D, Ohley W (1994) Fractal organization of trabecular bone images on calcaneus radiographs. J Bone Miner Res 9:1909-1918

5. Buckland-Wright JC, Lynch JA, Rymer J, Fogelman I (1994) Fractal signature analysis of macroradiographs measures trabecular organization in lumbar vertebrae of postmenopausal women. Calcif Tissue Int 54:106-112

6. Veenland JF, Grashuis JL, Van der Meer F, Beckers ALD, Gelsema ES (1996) Estimation of fractal dimension in radiographs. Med Phys 4:585-594

7. Mosekilde L, Mosekilde L, Danielsen CC (1987) Biomechanical competence of vertebral bone in relation to ash density and age in normal individuals. Bone 8:79-85

8. Hansson T, Roos B, Nachemson A (1980) The bone mineral content and ultimate compressive strength of lumbar vertebrae. Spine 5:46-55

9. Eriksson SAV, Isberg BO, Lindgren JU (1989) Prediction of vertebral strength by dual photon absorptiometry and quantitative computed tomography. Calcif Tissue Int 44:243-250

10. Myers BS, Arbogast KB, Lobaugh B, Harper KD, Richardson WJ, Drezner MK (1994) Improved assessment of lumbar vertebral body strength using supine lateral dual-energy $\mathrm{x}$-ray absorptiometry. J Bone Miner Res 9:687-693

11. Edmondston SJ, Singer KP, Day RE, Breidahl PD, Price RI (1994) In-vitro relationships between vertebral body density, size, and compressive strength in the elderly thoracolumbar spine. Clin Biomech 9:180-186

12. Moro M, Hecker AT, Bouxsein ML, Myers ER (1995) Failure load of thoracic vertebrae correlates with lumbar bone mineral density measured by DXA. Calcif Tissue Int 56:206-209

13. Singer K, Edmondston S, Day R, Breidahl P, Price R (1995) Prediction of thoracic and lumbar vertebral body compressive strength: correlations with bone mineral density and vertebral region. Bone 17:167-174

14. McBroom RJ, Hayes WC, Edwards WT, Goldberg RP, White III AA (1985) Prediction of vertebral body compressive fracture using quantitative computed tomography. J Bone Joint Surg Am 67:1206-1214
15. Lang SM, Moyle DD, Clemson D, Berg EW, Detorie N, Gilpin AT, Pappas NJ, Reynolds JC (1988) Correlation of mechanical properties of vertebral trabecular bone with equivalent mineral density as measured by computed tomography. J Bone Joint Surg Am 70-A:1531-1538

16. Brinckmann P, Biggemann M, Hilweg D (1989) Prediction of the compressive strength of human lumbar vertebrae. Clin Biomech 2:S1-S27

17. Mosekilde L, Bentzen SM, Ørtoft G, Jørgensen J (1989) The predictive value of quantitative computed tomography for vertebral body compressive strength and ash density. Bone 10: 465-470

18. Biggemann M, Hilweg D, Seidel S, Horst M, Brinckmann P (1991) Risk of vertebral insufficiency fractures in relation to compressive strength predicted by quantitative computed tomography. Eur J Radiol 13:6-10

19. Hayes WC, Piazza SJ, Zysset PK (1991) Biomechanics of fracture risk prediction of the hip and spine by quantitative computed tomography. Radiol Clin North Am 29:1-18

20. Mosekilde L, Mosekilde L (1988) Iliac crest trabecular bone volume as predictor for vertebral compressive strength, ash density and trabecular bone volume in normal individuals. Bone 9:195-199

21. Dempster DW, Ferguson-Pell MW, Mellish RWE, Cochran GVB, Xie F, Fey C, Parisien M, Lindsay R (1993) Relationships between bone structure in the iliac crest and bone structure and strength in the lumbar spine. Osteoporosis Int 3:9096

22. Caldwell CB, Willett K, Cuncins AV, Hearn TC (1995) Characterization of vertebral strength using digital radiographic analysis of bone structure. Med Phys 22:611-615

23. Haralick RM, Shanmugam K, Dinstein I (1973) Textural features for image classification. IEEE Trans Syst Man Cybern 3:610-621

24. Serra J (1982) Image analysis and mathematical morphology, 1 st ed. Academic Press, London

25. Veenland JF, Grashuis JL, Link TM, Gelsema ES (1997) Reproducibility of texture features in femur radiographs. Proceedings of international workshop "Trabecular bone texture analysis on radiographs," Orleans, France, December 18-20, 1996

26. Sachs L (1984) Angewandte statistik. Springer-Verlag, Berlin, Heidelberg

27. Nottestad SY, Baumel JJ, Kimmel DB, Recker RR, Heaney RP (1987) The proportion of trabecular bone in human vertebrae. J Bone Miner Res 2:221-229

28. Weszka JS, Dyer CR, Rosenfeld A (1976) A comparative study of texture measures for terrain classification. IEEE Trans Syst Man Cybern 6:269-285 